Research Article

\title{
Influence of Steering Angle Profiles on the Orbit Transfer Trajectory
}

\author{
Donghun Lee ${ }^{1}$ and Young-Joo Song $\mathbb{D D}^{2}$ \\ ${ }^{1}$ School of Aerospace and Mechanical Engineering, Korea Aerospace University, Goyang-si, Gyeonggi-do 10540, Republic of Korea \\ ${ }^{2}$ Lunar Exploration Program Office, Korea Aerospace Research Institute, Daejeon 34133, Republic of Korea \\ Correspondence should be addressed to Young-Joo Song; dear.yjsong@gmail.com
}

Received 19 August 2020; Revised 31 March 2021; Accepted 12 April 2021; Published 27 April 2021

Academic Editor: Adrian Neagu

Copyright (c) 2021 Donghun Lee and Young-Joo Song. This is an open access article distributed under the Creative Commons Attribution License, which permits unrestricted use, distribution, and reproduction in any medium, provided the original work is properly cited.

\begin{abstract}
This paper considers a planar orbit transfer trajectory design problem using finite thrust modeling. In this problem, the steering angles associated with the thrust direction are calculated from the predetermined profile format, and the unknown parameters in the profile are directly optimized. Three profile formats that were implemented in previous lunar exploration missions are considered. In addition, a steering angle profile defined in the rotating frame and the optimal steering angle profile are newly studied to compare the performances. To this end, the direct parameter optimization problem and the indirect optimization problem are formulated, and the characteristics of the steering angle profile and its influence on the transfer trajectory are analyzed.
\end{abstract}

\section{Introduction}

Numerous lunar exploration missions have been conducted to date [1-7], and the future missions are being planned by several space agencies and companies, such as Artemis (USA, NASA), Chandrayaan-3 (India, ISRO), SLIM (Japan, JAXA), and Luna 25 (Russia, Roscosmos) [8, 9]. In Korea, as a part of the Korean lunar exploration program, the Korea Pathfinder Lunar Orbiter (KPLO) is under development. In accordance with the KPLO mission requirements, the weak stability boundary (WSB)/ballistic lunar transfer (BLT) trajectories have been designed for transferring the KPLO spacecraft to the Moon $[10,11]$, and various research topics on the system design and operational strategies have been studied in this diverse field $[12,13]$.

In general, three mission phases may be considered before starting scientific missions on a lunar orbit: the trans-lunar cruise phase, the lunar orbit acquisition phase, and the commissioning phase [12]. Because a large amount of fuel is usually consumed during the lunar orbit acquisition phase, and the orbital maneuvers are the most critical events, appropriate orbit transfer strategies are necessary to achieve the scientifically predetermined mission orbit.

When developing the orbit transfer strategy for the lunar orbit acquisition phase, several factors should be taken into account. When the thrust-to-mass ratio is low, multiple orbital-maneuvers are included in the lunar orbit acquisition phase to reduce the gravitational loss. In the Lunar Reconnaissance Orbiter (LRO) or Selenological and Engineering Explorer (SELENE) mission, five or six sequences of orbital maneuvers were planned for the lunar orbit acquisition phase $[3,14]$. Moreover, the effects of perturbed accelerations due to the gravitational force of the Sun and the Earth on highly elliptical lunar orbits, which can be chosen as an intermediate orbit of the lunar orbit acquisition phase, are significant. These perturbed accelerations are related to the location of the periapsis and the Sun-Earth-Moon, geometric configuration; hence, this geometric configuration and the eccentricity vector at the lunar arrival time must also be considered, and the size of the intermediate orbit should be chosen carefully [4].

Furthermore, a finite thrust model, instead of an impulsive thrust model, is also necessary to reflect the effect of a 
relatively long maneuver duration. The transfer trajectory design problem using a finite thrust model involves finding the maneuver execution time, maneuver duration, and steering angle profile associated with the thrust direction command, and these features can be designed by solving a trajectory optimization problem.

The approaches for solving a trajectory optimization problem can be categorized into two methods: direct or indirect [15-22]. In the indirect method, the optimization problem is converted into a multipoint boundary value problem, and the optimal thrust magnitude and direction are determined by finding unknown state and costate variables. The solutions of the indirect method can guarantee optimality; however, an accurate initial guess of the unknown variables is necessary for the solution to converge. However, the indirect optimization is not easily applied to the problems in which complex perturbation forces are included [16]. Many advanced trajectory optimization technologies have been developed to overcome the drawbacks of the indirect or direct optimization method [23-26].

In the direct optimization method, the thrust magnitude and the steering angle profiles are parameterized by using an interpolation scheme or a specified function, and the unknown parameters in the profile are directly optimized using nonlinear programming [27]. In previous lunar exploration missions [2-5], the formats of the steering angles profiles were predetermined, and the unknown coefficients in the profile were optimized. In the Lunar Prospector mission [2], a single attitude was designed throughout the lunar orbit insertion maneuver sequence to minimize the operational complexity, which implies that a constant thrust direction in an inertial frame was designed for each orbital maneuver. In Reference [5], it was reported that the thrust direction was controlled by pitching the spacecraft attitude at a constant rate. This tactic implies that the thrust direction can be designed using a first-order polynomial in the inertial frame. In the LRO mission [4], the thrust direction command was designed to nearly coincide with the antivelocity direction to remove kinetic energy efficiently. When a transfer trajectory is designed using the antivelocity direction, the design parameters can be efficiently reduced because the thrust direction is determined from the velocity components.

In this paper, a trajectory design problem using finite thrust modeling is considered. The objective is to study the influence of the steering angle profile on the orbit transfer trajectory to develop a strategy for the lunar orbit acquisition phase. As discussed, several factors affect the transfer trajectories of the lunar orbit acquisition phase. To eliminate these other effects and to focus on the characteristics of each steering angle profiles, perturbation forces, such as the gravitational forces of the Sun and Earth and the solar radiation pressure, are excluded, and only planar orbital motions are considered. In this paper, the three steering angle profiles that were employed in previous lunar exploration missions are considered to design the transfer trajectories. In addition, the case in which the steering angle profile is defined in the rotating frame is newly studied, and the results are compared with those obtained with other approaches. To solve the direct optimization problem, an efficient method for guessing the unknown maneuver $\mathrm{du}$ ration and angular position is presented. Furthermore, the indirect optimization method is also formulated to find the optimal steering angle profiles and to compare the results. Three key contributions were made in this paper. The first is that a new two-point boundary value problem is formulated, where the equality constraints on the orbital specific energy and angular moment are considered. The second was that a linear steering angle profile in the rotating frame was newly investigated. The last was that characteristics of four steering-angle profiles were analyzed by the numerical studies for an orbit transfer problem under the finite thrust magnitude.

The remainder of this paper is organized as follows: First, the orbit transfer problem using a single finite-burn maneuver is formulated. Then, the indirect and direct optimization problems are formulated, and the design parameters for each steering angle profile are presented. The subsequent numerical examples explain the new findings associated with the influences of the steering angle profile on the orbit transfer trajectory design problem.

\section{Orbit Transfer Trajectory under a Single Finite-Burn Maneuver}

An example of an orbit transfer trajectory design problem is shown in Figure 1, where an initial orbit and a target orbit are given. In the problem, it is assumed that the periapsis altitudes of the initial and target orbits are close; therefore, there is a single orbital maneuver around the periapsis. The symbols $t^{s}$ and $t^{e}$ denote the start and end times of the finite-burn maneuver, and $t_{\text {duration }}$ is the maneuver duration, which is equivalent to $t^{e}-t^{s}$. The symbols $f^{s}$ and $f^{e}$ denote the true anomalies corresponding to $t^{s}$ and $t^{e}$, respectively.

The state variables are $x^{T}=\left[r, v_{r}, v_{\theta}, \theta, m\right]$ and are defined in Figure 2. In Figure 2, $X$ and $Y$ are the reference axes of the inertial frame; $m$ is the mass of the spacecraft, and $r$ and $\theta$ are a radial distance from the center of a central body to the spacecraft and an angular position, respectively; $v_{r}$ and $v_{\theta}$ are the radial and tangential velocity components, respectively; $T$ is the thrust magnitude, which is restricted to $0 \leq T \leq T_{\max }$ during the maneuver; and $\alpha$ is an in-plane steering angle defined in the rotating frame.

The system state dynamics in the rotating frame are provided in $[19,20]$

$$
\begin{aligned}
\dot{r} & =v_{r}, \\
\dot{v}_{r} & =\frac{v_{\theta}^{2}}{r}-\frac{\mu}{r^{2}}+\frac{T}{m} \sin (\alpha), \\
\dot{v}_{\theta} & =-\frac{v_{r} v_{\theta}}{r}+\frac{T}{m} \cos (\alpha), \\
\dot{\theta} & =\frac{v_{\theta}}{r},
\end{aligned}
$$




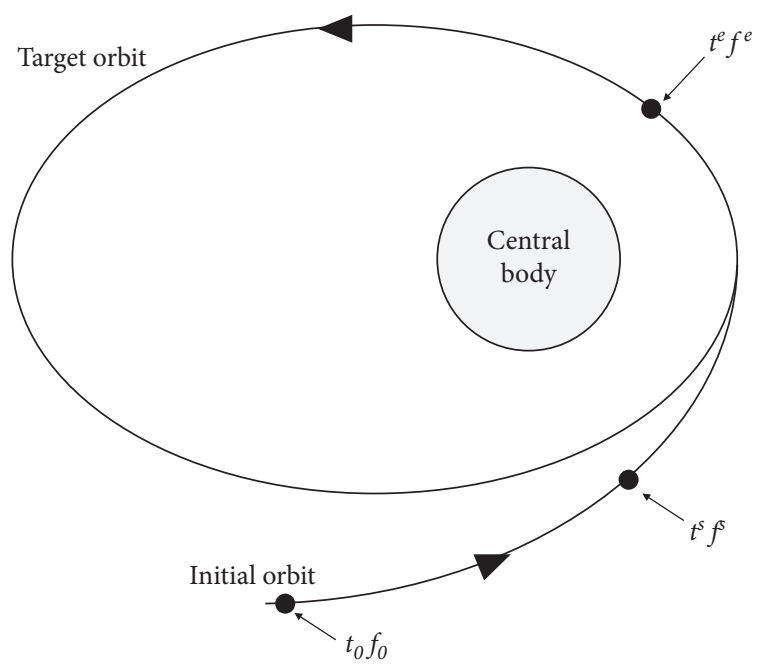

Figure 1: Illustration of the orbit transfer trajectory design problem.

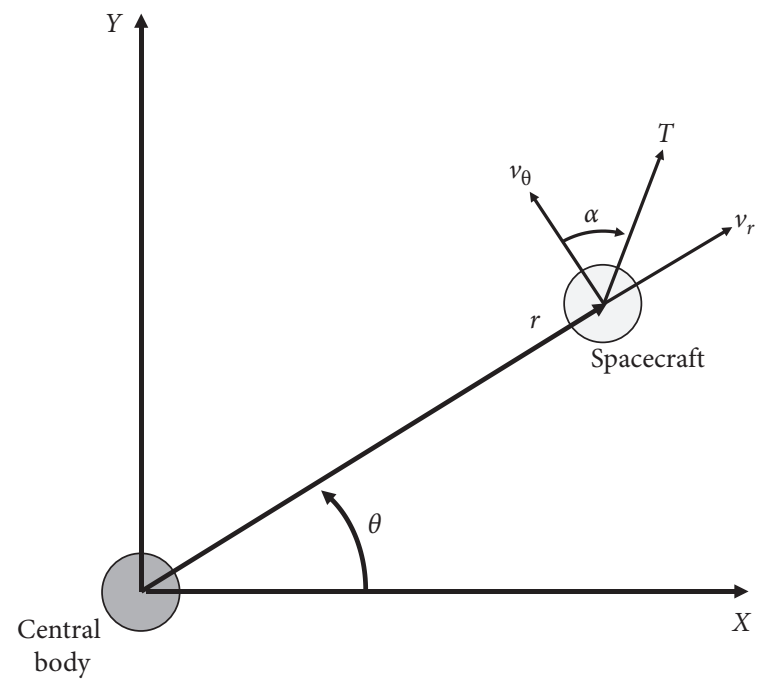

FIGURE 2: System state variables and an in-plane steering angle.

$$
\dot{m}=-\frac{T}{I_{\mathrm{SP}} g_{0}},
$$

where $g_{0}$ and $I_{\mathrm{SP}}$ denote Earth's gravitational acceleration and a constant specific impulse of the thruster, respectively. The objective is to minimize the amount of fuel consumption, which is equivalent to minimizing the maneuver duration when there is a single finite maneuver for the orbit transfer.

$$
J_{\text {main }}=t^{e}-t^{s}
$$

When a state vector $x$ is given at an epoch, the state vector can be transformed into two-dimensional, Keplerian orbital elements as follows [28]:

$$
\begin{aligned}
& a=\left[\frac{2}{r}-\frac{v_{r}^{2}+v_{\theta}^{2}}{\mu}\right]^{-1}, \\
& e=\sqrt{1-\frac{r^{2} v_{\theta}^{2}}{\mu a},} \\
& \omega=\theta-f, \\
& f=\left\{\begin{array}{l}
\text { if } v_{r}<0, \quad-\cos ^{-1}\left(\frac{r^{2} v_{\theta}^{2}}{\mu r e}-\frac{1}{e}\right) \mid, \\
\text { else, } \quad\left|\cos ^{-1}\left(\frac{r^{2} v_{\theta}^{2}}{\mu r e}-\frac{1}{e}\right)\right|,
\end{array}\right.
\end{aligned}
$$

where $a, e, \omega$, and $f$ are the semimajor axis, eccentricity, argument of the periapsis, and true anomaly, respectively. In Equations (7)-(10), the longitude of the ascending node is assumed to be zero. Inversely, when $a, e, \omega$, and $f$ are given at an epoch, the state variables can be calculated as follows [28]:

$$
\begin{aligned}
r & =\frac{a\left(1-e^{2}\right)}{1+e \cos (f)}, \\
v_{r} & =\sqrt{\frac{\mu}{p}} e \sin (f), \\
v_{\theta} & =\sqrt{\frac{\mu}{p}}(1+e \cos (f)), \\
\theta & =\omega+f .
\end{aligned}
$$

Let $\mathbf{O}_{0}=\left[a_{0}, e_{0}, \omega_{0}\right]^{T}$ and $\mathbf{O}_{f}=\left[a_{f}, e_{f}, \omega_{f}\right]^{T}$ be the orbital element vectors for the given initial orbit and final target orbit, respectively. If $f^{s}$ is given at $t^{s}$, the state vector at $t^{s}$ can be calculated from Equations (11)-(14), and the state boundary condition at the maneuver start time is specified as follows:

$$
\phi_{0}\left(t^{s}\right)=\left[\begin{array}{c}
r\left(t^{s}\right)-r\left(\theta^{s}-\omega^{s}\right) \\
v_{r}\left(t^{s}\right)-v_{r}\left(\theta^{s}-\omega^{s}\right) \\
v_{\theta}\left(t^{s}\right)-v_{\theta}\left(\theta^{s}-\omega^{s}\right) \\
m\left(t^{s}\right)-m_{0}
\end{array}\right] .
$$

In addition, the following boundary condition for the state variables at the maneuver end time is considered.

$$
\phi_{f}\left(t^{e}\right)=\left[\begin{array}{c}
\varepsilon\left(t^{e}\right)-\varepsilon_{t} \\
h\left(t^{e}\right)-h_{t}
\end{array}\right],
$$

where $\varepsilon=-\mu / r+\left(v_{r}^{2}+v_{\theta}^{2}\right) / 2=-\mu / 2 a$ is the specific orbital energy; $h=r v_{\theta}=\sqrt{\mu a\left(1-e^{2}\right)}$ is the angular momentum of an orbit; and $\varepsilon_{t}$ and $h_{t}$ are the target energy and angular momentum, respectively. Equation (16) implies that the 
semimajor axis and the eccentricity at the end of maneuver are the target values in the problem, whereas the argument of the periapsis is a free variable.

\section{Indirect Optimization Method}

The augmented cost function obtained by joining the boundary conditions in equations (15) and (16) with multipliers is as follows:

$$
\bar{J}=\rho_{0}^{T} \phi_{0}+\rho_{\varepsilon}\left[\varepsilon\left(t^{e}\right)-\varepsilon_{t}\right]+\rho_{h}\left[h\left(t^{e}\right)-h_{t}\right]+\int_{t^{s}}^{t^{e}} 1 \mathrm{~d} t .
$$

The Hamiltonian can be derived by applying a typical optimal control formulation, and the costate dynamics can be derived as follows:

$$
\begin{aligned}
& \dot{\lambda}_{r}=\lambda_{v r}\left(\frac{v_{\theta}^{2}}{r^{2}}-\frac{2 \mu}{r^{3}}\right)-\lambda_{v \theta}\left(\frac{v_{r} v_{\theta}}{r^{2}}\right)+\lambda_{\theta}\left(\frac{v_{\theta}}{r^{2}}\right), \\
& \dot{\lambda}_{v r}=-\lambda_{r}+\lambda_{v \theta}\left(\frac{v_{\theta}}{r}\right), \\
& \dot{\lambda}_{v \theta}=-2 \lambda_{v r}\left(\frac{v_{\theta}}{r}\right)+\lambda_{v \theta}\left(\frac{v_{\theta}}{r}\right)-\lambda_{\theta}\left(\frac{1}{r}\right), \\
& \dot{\lambda}_{\theta}=0,
\end{aligned}
$$

$$
\dot{\lambda}_{m}=\lambda_{v r}\left(\frac{T}{m^{2}} \sin \alpha\right)+\lambda_{v \theta}\left(\frac{T}{m^{2}} \cos \alpha\right) .
$$

The optimal control inputs for the in-plane steering angle and the thrust magnitude are derived as follows:

$$
\begin{aligned}
& \alpha=\tan ^{-1}\left(\frac{-\lambda_{v r}}{-\lambda_{v \theta}}\right), \\
& T= \begin{cases}T_{\max }, & \text { if } S<0, \\
T \in\left[0, T_{\max }\right], & \text { if } S=0, \\
0, & \text { if } S>0,\end{cases}
\end{aligned}
$$

where $S$ is the singular function as follows:

$$
S=-\frac{\sqrt{\lambda_{v r}^{2}+\lambda_{v \theta}^{2}}}{m}+\lambda_{m}\left(-\frac{1}{I_{\mathrm{SP}} g_{0}}\right) .
$$

Because the maneuver end time is free, the following equality constraint is imposed:

$$
H\left(t^{e}\right)=0 .
$$

The boundary conditions for the costate variables at $t^{s}$ and $t^{e}$ can be derived as follows:

$$
\begin{aligned}
& {\left[\begin{array}{c}
\lambda_{r}\left(t^{s}\right)-\rho_{r 0} \\
\lambda_{v r}\left(t^{s}\right)-\rho_{v r 0} \\
\lambda_{v \theta}\left(t^{s}\right)-\rho_{v \theta 0} \\
\lambda_{\theta}\left(t^{s}\right)-\left[\rho_{r 0}\left(-\frac{r^{2}}{p} e \sin (\theta-\omega)\right)+\rho_{v r 0}\left(-\sqrt{\frac{\mu}{p}} e \cos (\theta-\omega)\right)+\rho_{v \theta 0}\left(\sqrt{\frac{\mu}{p}} e \sin (\theta-\omega)\right)\right] \\
\lambda_{m}\left(t^{s}\right)-\rho_{m 0}
\end{array}\right]=0,} \\
& {\left[\begin{array}{c}
\lambda_{r}\left(t^{e}\right)-\rho_{\varepsilon} \frac{\mu}{r^{2}}-\rho_{h} v_{\theta} \\
\lambda_{v r}\left(t^{e}\right)-\rho_{\varepsilon} v_{r} \\
\lambda_{v \theta}\left(t^{e}\right)-\rho_{\varepsilon} v_{\theta}-\rho_{h} r \\
\lambda_{\theta}\left(t^{e}\right) \\
\lambda_{m}\left(t^{e}\right)
\end{array}\right]=0 .}
\end{aligned}
$$

Therefore, the orbit transfer trajectory design problem can be converted into a two-point boundary value problem. The unknown parameters are eight variables, which consist of the angular position at the beginning of the maneuver $\theta^{s}$; the maneuver duration $t_{\text {duration }}$; four initial costates $\lambda_{r}, \lambda_{v r}$, $\lambda_{v \theta}$, and $\lambda_{m}$; and two Lagrange multipliers $\rho_{\varepsilon}$ and $\rho_{h}$. The eight equality constraints are shown in equations (16), (26), and (28). 


\section{Direct Optimization Method}

Four options for the steering angle profile are considered in the direct optimization method: (1) a zero-order polynomial profile defined in the inertial coordinate frame, (2) a firstorder polynomial profile defined in the inertial coordinate frame, (3) a first-order polynomial profile defined in the rotating frame, and (4) the antivelocity direction along the trajectory. In the first option, the thrust direction is fixed in the inertial frame during the maneuver. The constant steering angle is chosen as the design parameter. In the second option, the steering angle in the inertial frame is calculated with a first-order polynomial profile, which implies that the thrust direction rotates at a constant angular velocity. In the third option, the steering angle in the rotating frame is calculated with a first-order polynomial profile, which implies that the rotational velocity of the thrust direction is not constant in the inertial frame. The thrust in the fourth option is in the anti-velocity direction, which can be calculated using the velocity components in the state variables. The mathematical expressions and numbers of unknown parameters for each steering angle profile are summarized in Table 1.

In Table 1, the superscript $I$ represents the inertial coordinate frame. The steering angle defined in the inertial frame can be transformed into that of the rotating frame by the following equation:

$$
\alpha=\theta+\frac{\pi}{2}-\alpha_{I}
$$

4.1. Initial Estimates of the Unknown Maneuver Duration. When solving the direct optimization problem, it is necessary to guess the unknown design parameters. To this end, an approach is proposed. First, the difference in the specific orbital energy between the initial and target orbits is calculated.

$$
\Delta \varepsilon=\varepsilon_{f}-\varepsilon_{0}
$$

where $\varepsilon_{0}$ and $\varepsilon_{f}$ are the specific orbital energies for the initial and target orbits, respectively. The change in the specific energy is also calculated using the state variables as follows:

$$
\Delta \varepsilon=\left(-\frac{\mu}{r_{f}^{\text {periapsis }}}+\frac{\left(v_{\theta f}^{\text {periapsis }}\right)^{2}}{2}\right)-\left(-\frac{\mu}{r_{0}^{\text {periapsis }}}+\frac{\left(v_{\theta 0}^{\text {periapsis }}\right)^{2}}{2}\right),
$$

where $r^{\text {periapsis }}$ and $v_{\theta}^{\text {periapsis }}$ are the radial distance and velocity of the periapsis, which are calculated using equations (11) and (13), respectively, with a radial velocity of zero at the periapsis. In equation (31), the subscripts 0 and $f$ denote the initial and the target orbits, respectively. From the assumption $r_{0}^{\text {periapsis }} \approx r_{f}^{\text {periapsis }}$, equation (31) can be rewritten as

$$
\Delta \varepsilon \approx \frac{\left(v_{\theta f}^{\text {periapsis }}\right)^{2}}{2}-\frac{\left(v_{\theta f}^{\text {periapsis }}-\Delta v^{\text {guess }}\right)^{2}}{2},
$$

where $\Delta v^{\text {guess }}$ is the estimated velocity increment from which the maneuver duration is calculated. From equations (30) and (32), $\Delta v^{\text {guess }}$ in equation (32) can be rewritten as follows:

$$
\Delta \nu^{\text {guess }}=v_{\theta f}^{\text {periapsis }}-\sqrt{-2\left(\varepsilon_{f}-\varepsilon_{0}\right)+\left(v_{\theta f}^{\text {periapsis }}\right)^{2}} \text {. }
$$

In addition, the estimated maneuver duration can be derived from the rocket equation using $\Delta v^{\text {guess }}$ in equation (33) as follows:

$$
t_{\text {duration }}^{\text {guess }}=\frac{I_{\mathrm{SP}} g_{0}}{T_{\max }} m_{0}-\left(\frac{\left.I_{\mathrm{SP}} g_{0} / T_{\max }\right) m_{0}}{\exp \left(\Delta V^{\text {guess }} / I_{\mathrm{SP}} g_{0}\right) .}\right.
$$

The true anomaly at the maneuver start time is estimated by solving Kepler equations. The Kepler equation for an elliptical orbit is given by [28]

$$
\sqrt{\frac{\mu}{a^{3}}}(t-\tau)=E-e \sin E,
$$

where $E$ is the eccentric anomaly, which has the following relationship with a true anomaly:

$$
\tan \frac{f}{2}=\sqrt{\frac{1+e}{1-e}} \tan \frac{E}{2}
$$

For a hyperbolic orbit, the hyperbolic Kepler equation is given by [28]

$$
\sqrt{\frac{\mu}{(-a)^{3}}}(t-\tau)=e \sinh H-H
$$

where $H$ is the hyperbolic eccentric anomaly, which has the following relationship with a true anomaly $f$ :

$$
\tan \frac{1}{2} f=\sqrt{\frac{e+1}{e-1}} \tanh \frac{1}{2} H .
$$

Let $f_{0}^{*}$ and $f_{f}^{*}$ be the true anomalies, which are calculated from equations (35) and (36) or equations (37) and (38) using the initial orbit element and final orbit element with $t=t_{\text {duration }}^{\text {guess }}$ and $\tau=0$, respectively. The estimated true anomaly at the beginning of the maneuver is calculated using $f_{0}^{*}$ and $f_{f}^{*}$ as follows:

$$
\left(f^{s}\right)^{\text {guess }}=-\left|\frac{f_{0}^{*}+f_{f}^{*}}{2}\right| .
$$

\section{Numerical Studies}

In this section, numerical examples are presented for the orbit transfer trajectory design problem using a finite-burn maneuver. The constants used in the numerical examples are presented in Table 2. The central body is the Moon, and the lunar mean radius and gravitational constant are $1737.4 \mathrm{~km}$ and $4902.8 \mathrm{~km}^{3} / \mathrm{s}^{2}$, respectively. The specific impulse and maximum thrust magnitude are assumed to be 227 seconds and $120 \mathrm{~N}$, respectively.

In the first example, the initial and target orbits are a hyperbolic orbit and an elliptical orbit, respectively, of which 
TABLE 1: Options for the steering angle profiles.

\begin{tabular}{lcc}
\hline Option & Steering angle profile & Number of design parameters \\
\hline (1) Zero order (inertial frame) & $\alpha^{I}(t)=\alpha^{0}, t \in\left[t^{s}, t^{e}\right]$ & $3\left(\alpha^{0}, \theta^{s}, t_{\text {duration }}\right)$ \\
(2) $1^{\text {st }}$-order polynomial (inertial frame) & $\alpha^{I}(t)=\alpha^{1}\left(t-t^{s}\right)+\alpha^{0}, t \in\left[t^{s}, t^{e}\right]$ & $4\left(\alpha^{0}, \alpha^{1}, \theta^{s}, t_{\text {duration }}\right)$ \\
(3) $1^{\text {st }}$-order polynomial (rotating frame) & $\alpha(t)=\alpha^{1}\left(t-t^{s}\right)+\alpha^{0}, t \in\left[t^{s}, t^{e}\right]$ & $4\left(\alpha^{0}, \alpha^{1}, \theta^{s}, t_{\text {duration }}\right)$ \\
(4) Antivelocity direction & $\alpha(t)=\tan ^{-1}\left(v_{r}(t) / v_{\theta}(t)\right), t \in\left[t^{s}, t^{e}\right]$ & $2\left(\theta^{s}, t_{\text {duration }}\right)$ \\
\hline
\end{tabular}

TABLE 2: Constants in the numerical examples.

\begin{tabular}{lcc}
\hline & Notation & Value \\
\hline Gravity constant of the Moon & $\mu$ & $4902.8 \mathrm{~km}^{3} / \mathrm{s}^{2}$ \\
Mean radius of the Moon & $R_{\text {Moon }}$ & $1737.4 \mathrm{~km}$ \\
Earth's gravitational acceleration & $g_{0}$ & $9.807 \mathrm{~m} / \mathrm{s}^{2}$ \\
Maximum thrust magnitude & $T_{\max }$ & $120 \mathrm{~N}$ \\
Specific impulse of the thruster & $I_{\mathrm{SP}}$ & $227 \mathrm{~second}$ \\
Initial mass & $m_{0}$ & $678 \mathrm{~kg}$ \\
\hline
\end{tabular}

the Keplerian orbital elements are summarized in Table 3. The periapsis altitude of the initial orbit and target orbit is chosen as $200 \mathrm{~km}$.

The TPBVP of the indirect method in Section 3 was solved by the conventional single shooting method. In the problem, the initial guess for the unknown maneuver duration and true anomaly at the maneuver start time was calculated by equations (34) and (39). For the unknown initial costate values, we tested several initial values for the first example below. In the second example, a discrete continuation method was applied, where the periapsis altitude was the discretized parameter for the continuation method [26]. The initial estimates of the maneuver duration and the angular position at the beginning of the maneuver were calculated from equations (34) and (39) as 2281.97 seconds and $-65.48^{\circ}$, respectively. Figure 3 shows the transfer trajectory optimized by the indirect optimization method. The true anomaly at the maneuver start time, the maneuver duration, and the fuel consumption were approximately $-72.2186^{\circ}, 2510.3 \mathrm{~s}$, and $135.3193 \mathrm{~kg}$, respectively. These results are summarized in Table 4. The Hamiltonian of the indirect method is presented in Figure 4 to check the optimality of the result. In Figure 4, zero seconds are the maneuver start time. As shown in Figure 4, the Hamiltonian is zero throughout the maneuver phase, which implies that the solution is a local minimum. The optimal steering angle profile is represented by a solid black line in Figure 5.

The orbit transfer problem was also solved by the direct method, for which the four options for the steering angle profile in Table 1 were applied. The direct optimization problem was solved by sequential quadratic programming in MATLAB software. The transfer trajectory designed by using the zero-order polynomial profile (option 1) is presented in Figure 6. The solutions related to the unknown design parameters for all the options in Table 1 are summarized in Table 4. As shown in Table 4, when the transfer trajectory was designed by using the first-order polynomial profile defined in the rotating frame (option 3), the performance was most efficient in terms of fuel consumption.
TABLE 3: Orbit parameters for the initial and final orbits (Example 1).

\begin{tabular}{lcc}
\hline & Initial orbit & Target orbit \\
\hline Semimajor axis & $-7341.7191 \mathrm{~km}$ & $3869.5815 \mathrm{~km}$ \\
Eccentricity & 1.2639 & 0.4993 \\
Argument of the periapsis & 0 degree & Free \\
Periapsis altitude & $200 \mathrm{~km}$ & $200 \mathrm{~km}$ \\
Orbital period & - & 6.0 hours \\
\hline
\end{tabular}

Furthermore, the result was closest to that of the indirect method, which can be understood from Figure 5 .

Figure 5 shows the steering angle profiles represented in the inertial frame. In Figure 5, the steering angle calculated in the rotating frame was transformed into that of the inertial frame using equation (29). As shown in Figure 5, it can be observed that the first-order polynomial profile defined in the rotating frame (option 3 , blue line) was most suitable for approximating the optimal steering angle profile (indirect method, black line); however, even though the first-order polynomial profile defined in the inertial frame (option 2, green line) appears to differ from the optimal steering angle profile, it was observed that the firstorder polynomial profile in the inertial frame could also approximate the optimal steering angle profile efficiently. As listed in Table 4, the difference with the indirect method in terms of fuel consumption was approximately $0.0047 \mathrm{~kg}$, $0.26 \mathrm{~kg}$, and $3.9 \mathrm{~kg}$ for options 3,2 , and 1 , respectively. This fact indicates that the first-order polynomial profiles defined in the inertial and rotating frames are more appropriate in this example. It is important to note that there was no solution when the steering angle profile was the antivelocity direction (option 4).

In Figure 7, the time histories of the instantaneous argument of the periapsis for the transfer trajectories are presented. Unlike the results of the indirect method and options 2 and 3, the instantaneous argument of the periapsis for the zero-order polynomial profile (option 1) was gradually increased until the end of the maneuver. In this example, the argument of the periapsis at the end of the maneuver was approximately $10^{\circ}$ for option 1 , whereas it was less than $0.5^{\circ}$ for the indirect method and options 2 and 3 .

In Figure 8, the instantaneous periapsis altitudes of the transfer trajectories are presented for all the steering angle profile options except option 4. For the indirect method and both first-order polynomial profiles (options 2 and 3), the instantaneous periapsis altitude was increased at the beginning of the maneuver and monotonically decreased to the target value approximately after 0.15 hours; however, the instantaneous periapsis altitude was decreased below the 


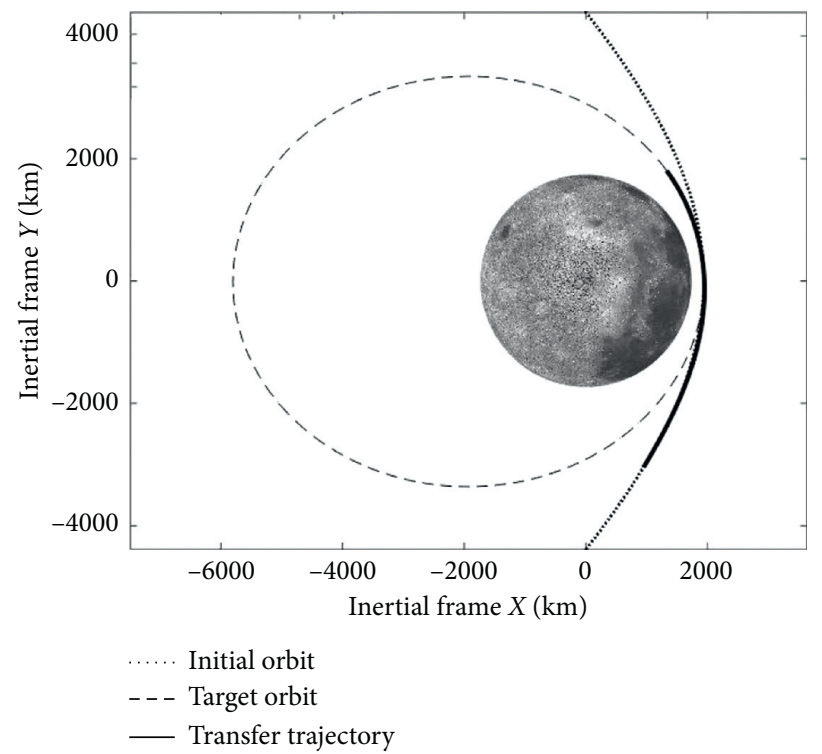

FIGURE 3: Initial hyperbolic orbit, final target orbit, and transfer trajectory, (Case: Indirect optimization method.)

TABLE 4: Results for the example in Table 3.

\begin{tabular}{lccc}
\hline Options of the steering angle profile & $f^{s}$ (degrees) & $t_{\text {duration }}($ seconds $)$ & $\Delta m^{\text {feul }}(\mathrm{kg})$ \\
\hline Indirect method & -72.2186 & 2510.3865 & 135.3193 \\
Option 1. zero order(inertial frame) & -76.9592 & 2582.4696 & 139.2049 \\
Option 2. $1^{\text {st }}$-order polynomial(inertial frame) & -71.7423 & 2515.1796 & 135.5777 \\
Option 3. $1^{\text {st }}$-order polynomial(rotating frame) & -72.2090 & 2510.4736 & 135.3240 \\
Option 4. Antivelocity direction & Not converged & Not converged & Not converged \\
\hline
\end{tabular}

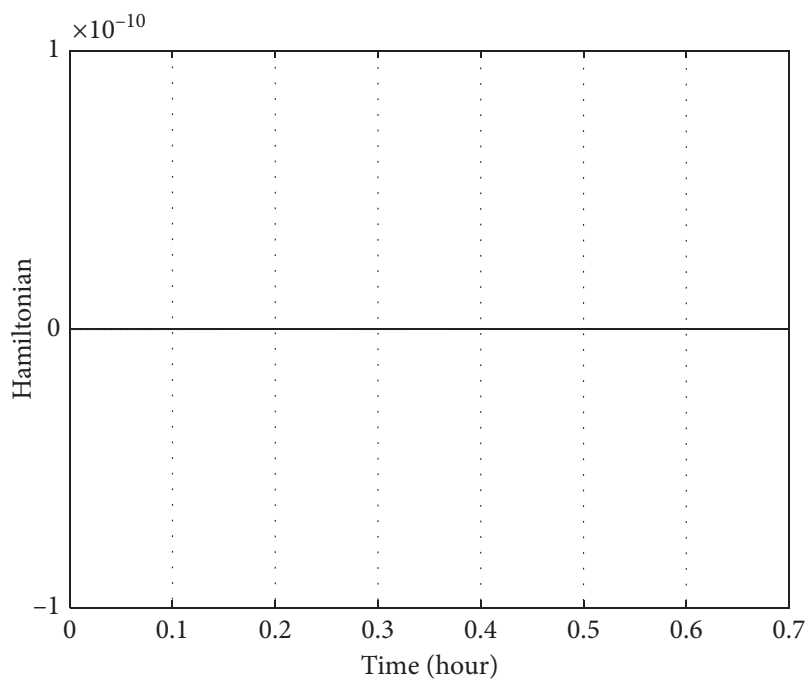

FIGURE 4: Hamiltonian (result of the indirect optimization method).

periapsis altitude of the target orbit in the case of option 1 , and then it increased to the target value after approximately 0.53 hours. This fact implies that there is a proper periapsis altitude for the initial orbit under the same orbital energy, which can reduce the amount of fuel consumption for the transfer.

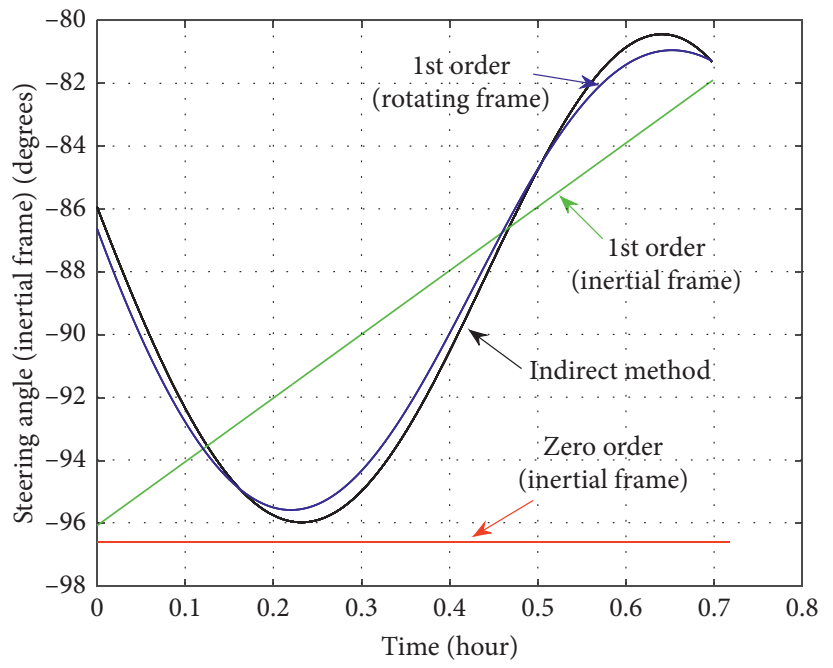

Figure 5: Steering angle profile.

In the second example, the boundary conditions, which are summarized in Table 5, were identical to those of Example 1 except for the periapsis altitude of the initial hyperbolic orbit. The specific orbital energy of the initial orbit was fixed; however, the periapsis altitude of the initial orbit was varied from 150 to $350 \mathrm{~km}$. The periapsis altitude of the final target orbit was $200 \mathrm{~km}$, and the argument of the 


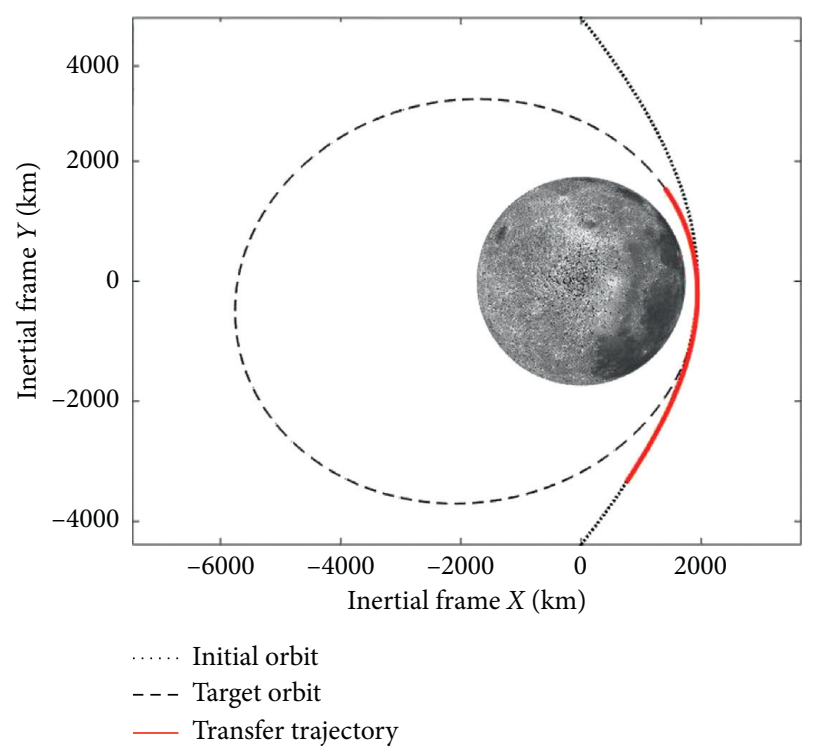

FIGURE 6: Initial hyperbolic orbit, final target orbit, and transfer trajectory (case: zero-order polynomial profile in the inertial frame).

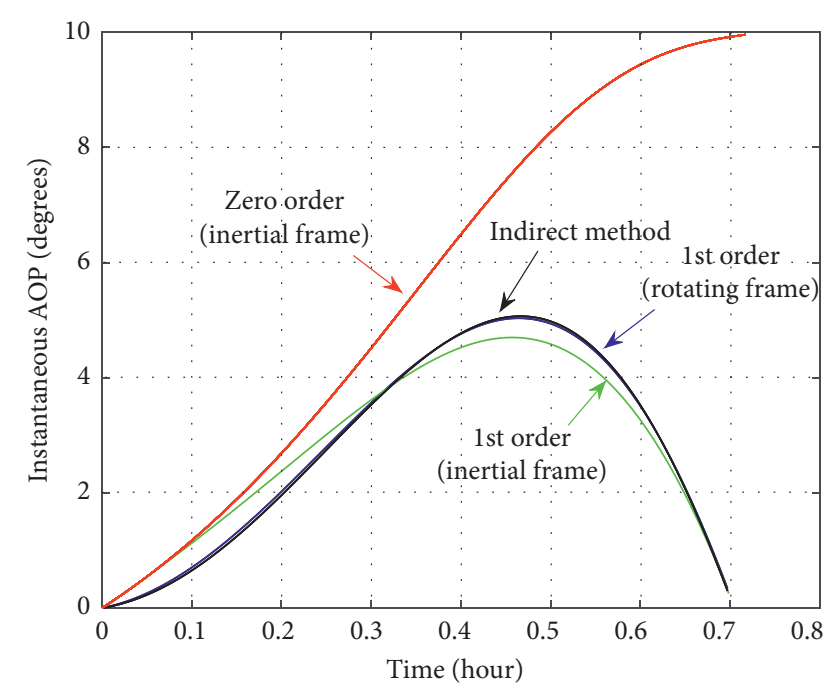

FIgURE 7: Instantaneous argument of periapsis.

periapsis was a free variable. The solutions in example 1 were used as the initial guess for example 2. The discrete continuation method was applied to find other problems where the periapsis altitude was varied from $200 \mathrm{~km}$. The convergence tolerance was $10^{-12}$, and every solution in the continuation method was converged to the solution within 20 iterations.

The transfer trajectory design problems were solved by using an indirect or direct optimization method. Figure 9 shows the variations in the fuel consumption according to the periapsis altitude of the initial orbit. As shown in Figure 9, a minimum point was found for each steering angle profile option. First, the trends in the fuel consumption were similar for the indirect method and both first-order polynomials (options 2 and 3). The minimum points were within

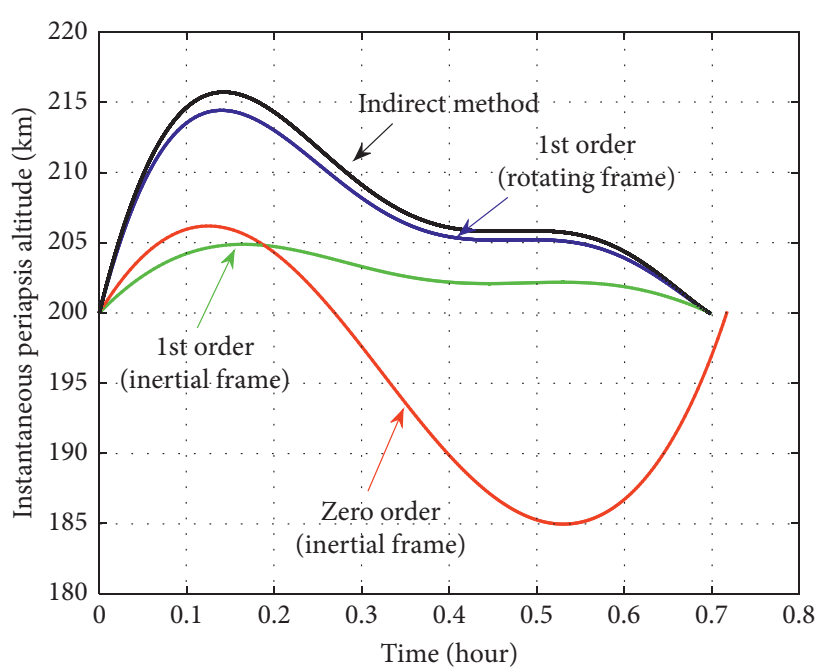

FIgURE 8: Instantaneous periapsis altitude.

TABle 5: Boundary conditions for the initial and final orbits (Example 2).

\begin{tabular}{lcc}
\hline & Initial orbit & Target orbit \\
\hline Semimajor axis & $-7341.7191 \mathrm{~km}$ & $3869.5815 \mathrm{~km}$ \\
Eccentricity & $1.2571 \sim 1.2843$ & 0.4993 \\
Argument of the periapsis & 0 degree & Free \\
Periapsis altitude & $150 \sim 350 \mathrm{~km}$ & $200 \mathrm{~km}$ \\
Orbital period & - & 6.0 hour \\
\hline
\end{tabular}

$297.5 \pm 0.5 \mathrm{~km}$ for the indirect method and both first-order polynomials (options 2 and 3). These values are higher than those of the final orbit. This result implies that when an orbital maneuver is executed using a first-order polynomial profile to change the orbital energy and angular momentum, it is more desirable to execute the orbital maneuver from the orbit in which the periapsis altitude is higher than that of the target orbit. If there are multiple maneuvers near the periapsis, the overall transfer trajectory is a spiral motion.

In the case of the zero-order polynomial profile (option 1), when the periapsis altitude of the initial orbit was approximately $173 \mathrm{~km}$, which was lower than the $200 \mathrm{~km}$ periapsis altitude of the final orbit, the fuel consumption was minimal. Therefore, when an orbital maneuver is executed using a zeroorder polynomial profile to change the orbital energy and angular momentum, it is more desirable to execute the orbital maneuver from the orbit in which periapsis altitude is below that of the target orbit. This result is contrary to the findings for the first-order polynomial profiles.

For option 4, solutions were found when the periapsis altitude of the initial hyperbolic orbit exceeded approximately $305.15 \mathrm{~km}$, and the minimum point of the periapsis altitude was approximately $305.15 \mathrm{~km}$. The fuel consumption increased linearly with the periapsis altitude of the initial hyperbolic orbit.

Figure 10 shows the differences in fuel consumption between the indirect method and the other cases. The fuel consumption performances of option 2 and particularly 


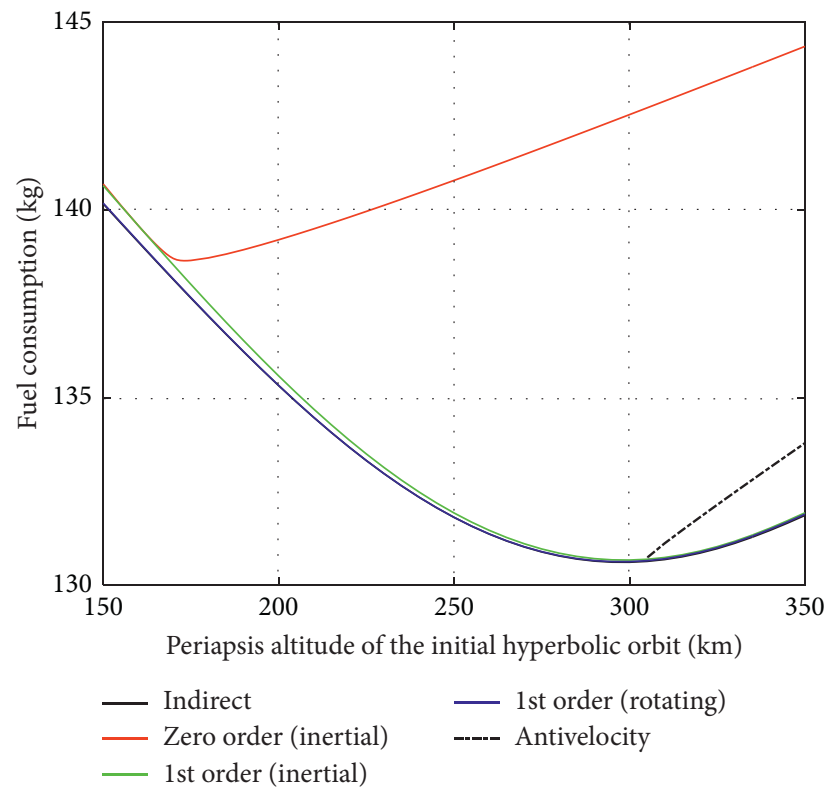

Figure 9: Plot of the fuel consumptions against the periapsis altitude.

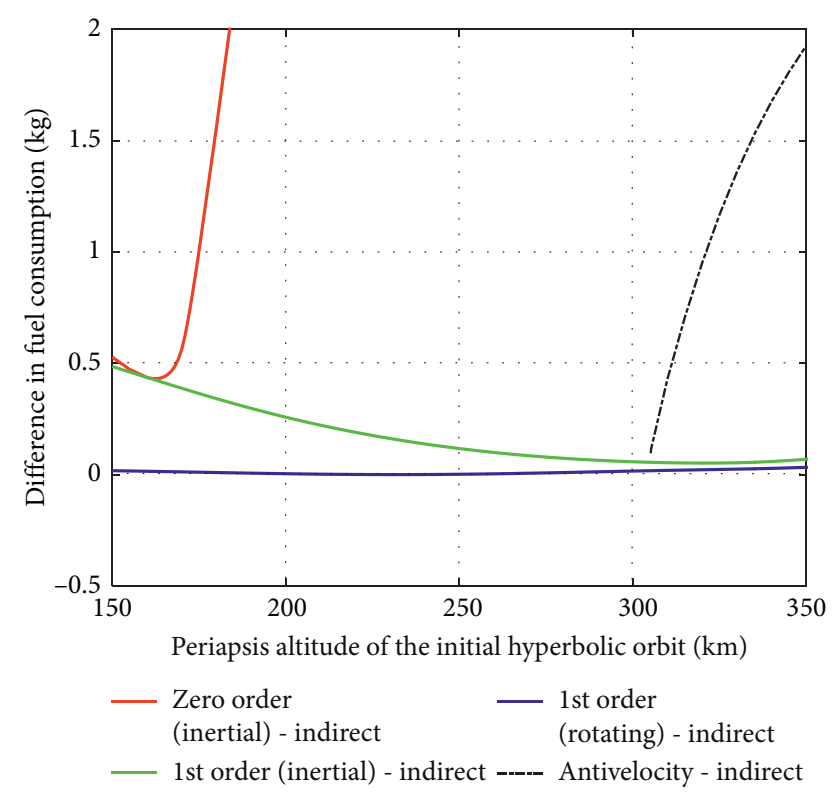

FiguRe 10: Differences in fuel consumption.

option 3 were close to that of the indirect method. For option 1 , the difference decreased until approximately $162 \mathrm{~km}$ periapsis altitude of the initial orbit and then increased drastically.

Figure 11 shows the variation in the argument of the periapsis at the maneuver end time. For the indirect method and both first-order polynomial profiles (options 2 and 3), the argument of the periapsis was less than $1.5^{\circ}$ when the periapsis altitude was below approximately $300 \mathrm{~km}$. For the zero-order polynomial profile (option 1) and the anti-velocity direction (option 4), the amount of variation was more drastic than the amounts for the other cases.

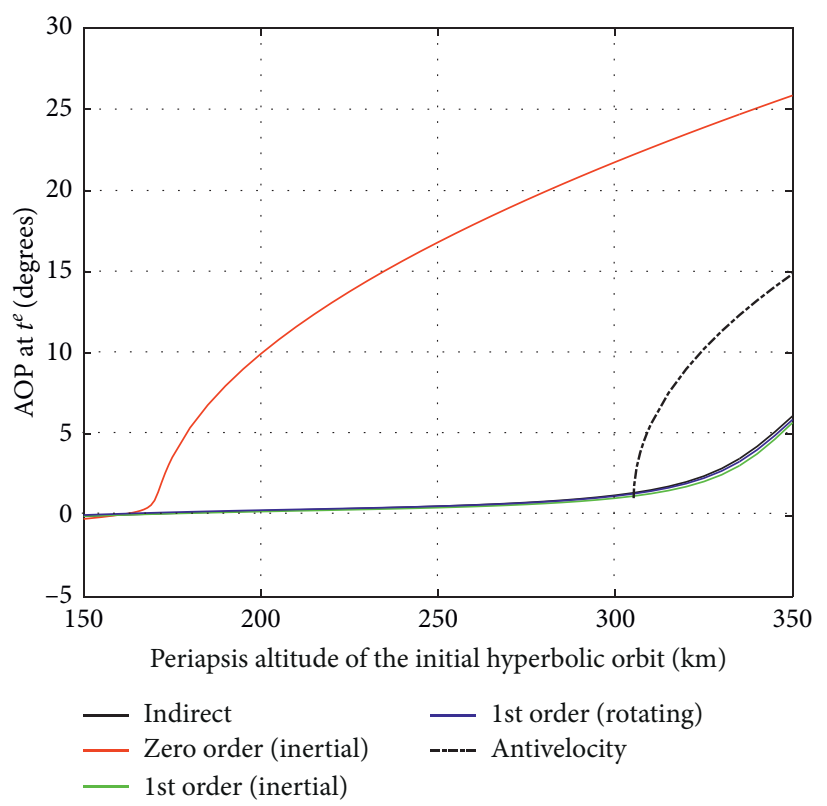

FIgURE 11: Variation in the argument of the periapsis at the end of the maneuver.

In Figure 12, the variations in the steering angle profiles according to the initial periapsis altitude are presented. As shown in Figure 12(a), the constant steering angle for the zero-order polynomial profile (option 1) decreased from approximately $-87^{\circ}$ to $-106.35^{\circ}$ as the periapsis altitude was increased. For option 2, the steering angle at the maneuver start time decreased from approximately $-88^{\circ}$ to $-140^{\circ}$ as the initial periapsis altitude was increased, while the slope of the steering angle profile was increased. When the periapsis altitude was approximately $159.6 \mathrm{~km}$, the slope was zero; therefore, the amount of fuel consumption for option 2 was 


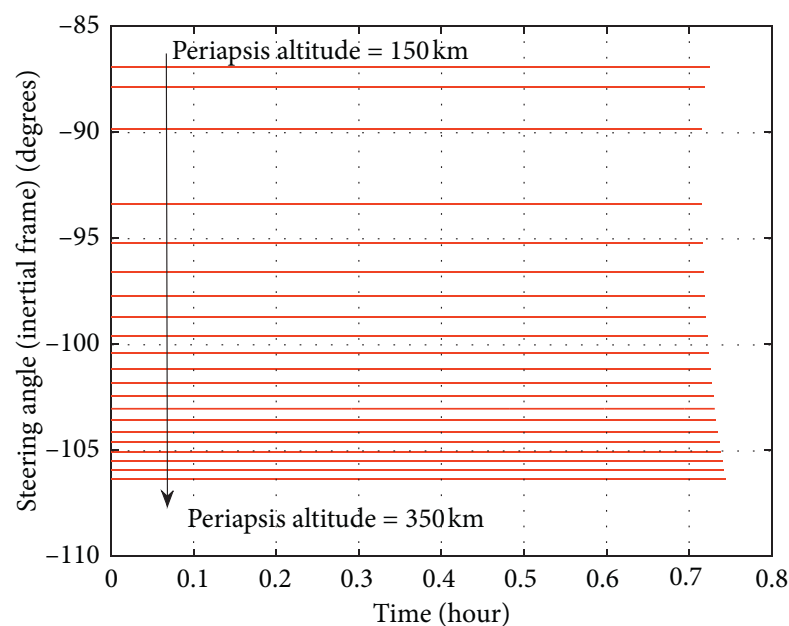

(a)

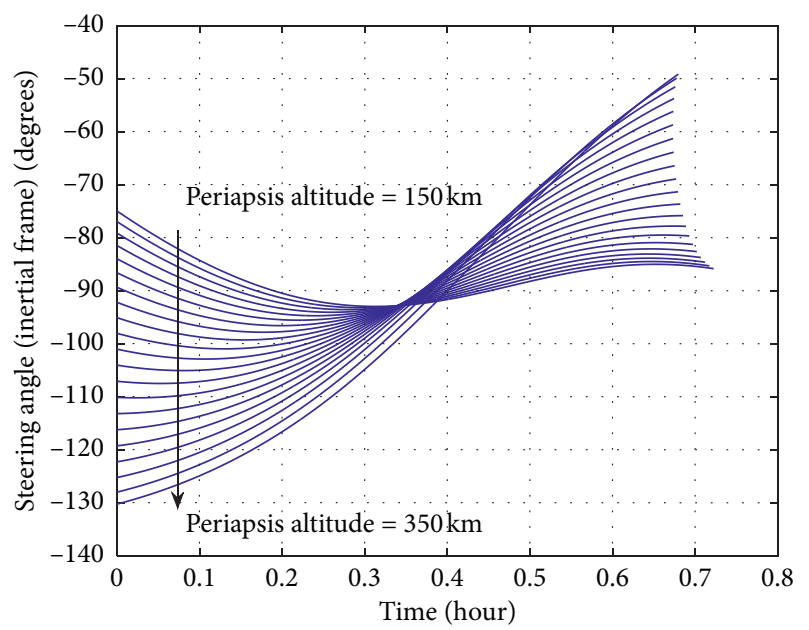

(c)

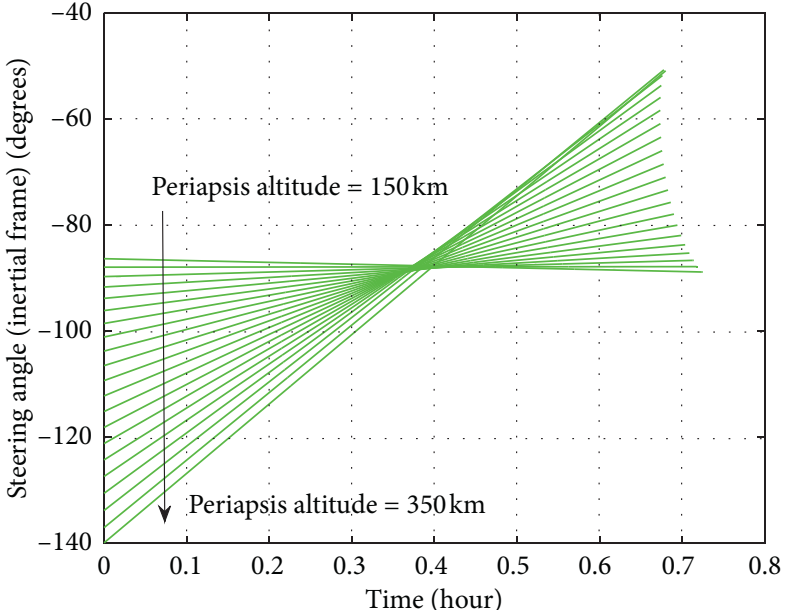

(b)

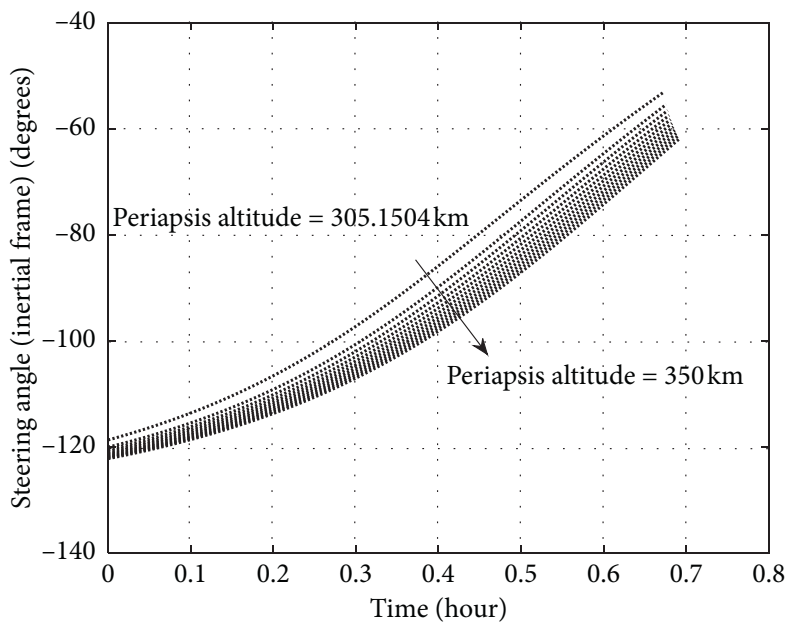

(d)

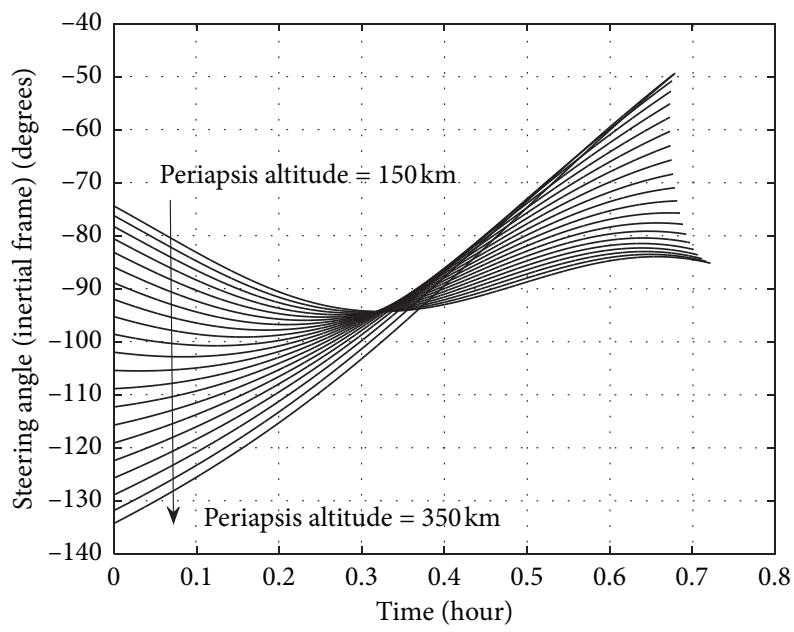

(e)

FIgURE 12: Variation in the steering angle profiles according to the initial periapsis altitude. (a) Option 1: zero order (inertial frame), (b) option 2: 1st order (inertial frame), (c) option 3: 1st order (rotating frame), (d) option 4: antivelocity direction, and (e) the indirect method. 
identical to that for option 1 , which can also be observed in Figures 9 and 10. In the case of option 3, the variation in the steering angle profile was the closest to the variation observed with the indirect method. Therefore, even though the periapsis altitude of the initial orbit was $150 \mathrm{~km}$, the difference in the fuel consumption for option 3 was not significant as shown in Figure 10.

\section{Conclusions}

In this paper, the orbit transfer problem from an initial hyperbolic orbit to an elliptical orbit was studied. The indirect optimization problem was formulated, where the terminal constraints were the orbital specific energy and angular momentum. The direct parameter optimization problem was also formulated, where the unknown parameters were the angular position at the beginning of the maneuver, the maneuver duration, and the parameters in the steering angle profiles. An initial estimation method for the angular position and maneuver duration was presented to solve the direct optimization problem. In the direct optimization problem, four steering angle profiles were considered, namely, a zero-order polynomial in the inertial frame, a first-order polynomial in the inertial frame, a firstorder polynomial in the rotating frame, and the antivelocity direction. Through numerical examples, the trends in fuel consumption, the argument of the periapsis, and the periapsis altitude were studied. Through these numerical studies, it was observed that when the orbit transfer is executed using the zero-order polynomial profile, a periapsis altitude of the initial orbit lower than that of the final orbit shows better results in terms of fuel consumption and the variation in the argument of the periapsis. In contrary, when the orbit transfer is executed using the first-order polynomial profile or the antivelocity profile, starting the orbit transfer from a periapsis altitude of the initial orbit higher than that of the final orbit shows better results.

\section{Data Availability}

The data used to support this study are available from the corresponding author upon request.

\section{Conflicts of Interest}

The authors declare that there are no conflicts of interest regarding the publication of this paper.

\section{Acknowledgments}

This research was supported by the Korea Aerospace University.

\section{References}

[1] T. C. Sorensen and P. D. Spudis, "The Clementine mission-A 10-year perspective," Journal of Earth System Science, vol. 114, no. 6, pp. 645-668, 2005.

[2] D. Lozier, K. Galal, D. Folta, and M. Beckman, "Lunar prospector mission design and trajectory support," in
Proceedings of the AAS/GSFC International Symposium on Space Flight Dynamics, Greenbelt, MD, USA, May 1998.

[3] S. Matsumoto, Y. Takizawa, M. Ogawa et al., "Flight trajectory and control system of SELenological and engineering explorer "KAGUYA" mission to the moon," in Proceedings of the AIAA Guidance, Navigation and Control Conference and Exhibit, Honolulu, HI, USA, August 2008.

[4] J. E. Sedlak and M. B. Houghton, "Lunar reconnaissance orbiter (LRO) attitude maneuver planning," in Proceedings of the International Symposium on Spaceflight Dynamics, Toulouse, France, May 2009.

[5] J. Beerer and G. G. Havens, "Operating the dual-orbiter GRAIL mission," in Proceedings of the SpaceOps, Stockholm, Sweden, June 2012.

[6] V. Sundararajan, "Indian lunar space exploration program Chandrayaan I and II missions," in Proceedings of the AIAA SPACE 2012 Conference \& Exposition, Pasadena, CA, USA, September 2012.

[7] M. Loucks, L. Plice, D. Cheke, C. Maunder, and B. Reich, "The LADEE trajectory as flown," in Proceedings of the AAS/AIAA Space Flight Mechanics Meeting, Williamsbug, VA, USA, January 2015.

[8] T. H. Sweetser, S. B. Broschart, V. Angelopoulos et al., "ARTEMIS mission design," Space Science Reviews, vol. 165, no. 1-4, pp. 27-57, 2011.

[9] S.-I. Sakai and S. Sawai, "Introduction of SLIM, a small and pinpoint lunar lander," in Proceedings of the Japan Geoscience Union Meeting, Yokohama, Japan, May 2014.

[10] D. Lee, J.-I. Park, Y.-J. Song et al., "KPLO BLT trajectory design," in Proceeding of the 2020 KSAS Spring Conference, Goseung, Republic of Korea, July 2020.

[11] S. Hong, Y.-R. Kim, Y.-J. Song et al., "Launch vehicle dispersion analysis of KPLO BLT trajectory," in Proceeding of the 2020 KSAS Spring Conference, Goseung, Republic of Korea, July 2020.

[12] J.-I. Park, D. Lee, Y.-R. Kim et al., "Lunar orbit insertion and mission orbit design for KPLO BLT trajectory," in Proceeding of the 2020 KSAS Spring Conference, Goseung, Republic of Korea, July 2020.

[13] Y.-R. Kim, Y.-J. Song, J.-I. Park et al., "Orbit determination and prediction simulation of KPLO BLT trajectory," in Proceeding of the 2020 KSAS Spring Conference, Goseung, Republic of Korea, July 2020.

[14] M. Mesarch, M. Beckman, D. Folta, R. Lamb, and K. Richon, "Maneuver operations results from the lunar reconnaissance orbiter (LRO) mission," in Proceedings of the SpaceOps 2010 conference, Huntsville, Alabama, April 2010.

[15] Y.-J. Song, J. Woo, S.-Y. Park, K.-H. Choi, and E.-S. Sim, "The earth-moon transfer trajectory design and analysis using intermediate loop orbits," Journal of Astronomy and Space Sciences, vol. 26, no. 2, pp. 171-186, 2009.

[16] Y.-J. Song, S.-Y. Park, H.-D. Kim, J.-H. Lee, and E.-S. Sim, "Analysis of delta-V losses during lunar Capture sequence using finite thrust," Journal of Astronomy and Space Sciences, vol. 28, no. 3, pp. 203-216, 2011.

[17] C. A. Ocampo and R. Mathur, "Variational model for optimization of finite-burn escape trajectories using a direct method," Journal of Guidance, Control, and Dynamics, vol. 35, no. 2, pp. 598-608, 2012.

[18] C. Ocampo and J.-P. Munoz, "Variational equations for a generalized spacecraft trajectory model," Journal of Guidance, Control, and Dynamics, vol. 33, no. 5, pp. 1615-1622, 2010.

[19] D. Lee and H. Bang, "Efficient initial costates estimation for optimal spiral orbit transfer trajectories design," Journal of 
Guidance, Control, and Dynamics, vol. 32, no. 6, pp. 19431947, 2009.

[20] C. L. Ranieri and C. A. Ocampo, "Indirect optimization of spiral trajectories," Journal of Guidance, Control, and Dynamics, vol. 29, no. 6, pp. 1360-1366, 2006.

[21] D. G. Hull, Optimal Control Theory for Applications, Springer, New York, NY, USA, 2003.

[22] H.-X. Shen, Z.-S. Duan, and L. Casalino, "Optimal thrust control with magnitude and direction constraints," Acta Astronautica, vol. 162, pp. 417-423, 2019.

[23] H. Peng, Q. Gao, Z. Wu, and W. Zhong, "Symplectic approaches for solving two-point boundary-value problems," Journal of Guidance, Control, and Dynamics, vol. 35, no. 2, pp. 653-659, 2012.

[24] X. Wang, H. Peng, S. Zhang, B. Chen, and W. Zhong, "A symplectic pseudospectral method for nonlinear optimal control problems with inequality constraints," ISA Transactions, vol. 68, pp. 335-352, 2017.

[25] H. J. Peng, Q. Gao, Z. G. Wu, and W. X. Zhong, "Symplectic adaptive algorithm for solving nonlinear two-point boundary value problems in astrodynamics," Celestial Mechanics and Dynamical Astronomy, vol. 110, no. 4, pp. 319-342, 2011.

[26] D. Lee and Y.-J. Song, "Spin-to-spin slew maneuvers under spherically constrained angular acceleration," Advances in Space Research, vol. 64, no. 6, pp. 1274-1285, 2019.

[27] D. Lee, "Orbit transfer problems under a thrust vector constraint," in Proceeding of the 2019 KSAS Fall Conference, Jeju, Republic of Korea, November 2019.

[28] R. H. Battin, "An introduction to the mathematics and methods of astrodynamics, revised edition," AIAA Education Series, American Institute of Aeronautics and Astronautics, NewYork, NY, USA, 1999. 